\title{
GENERALIZED HANSEN COEFFICIENTS
}

\author{
SŁAWOMIR BREITER ${ }^{1}$, GILLES MÉTRIS ${ }^{2}$ and DAVID VOKROUHLICKÝ ${ }^{3}$ \\ ${ }^{1}$ Astronomical Observatory of A, Mickiewicz University, Stoneczna 36, \\ PL60-286 Poznań, Poland, e-mail: breiter@amu.edu.pl \\ ${ }^{2}$ Dept. CERGA, Observatoire de la Côte d'Azur, Av. N. Copernic, F 06130 Grasse, \\ France,e-mail: gilles.metris@obs-azur.fr \\ ${ }^{3}$ Institute of Astronomy, Charles University, V Holešovičkách 2, CZ 18000 Prague 8, \\ Czech Republic, e-mail:vvokrouhl@cesnet.cz.
}

(Received: July 2003; revised: 11 August 2003; accepted: 18 August 2003)

\begin{abstract}
Hansen coefficients $X_{k}^{n, j}$ were originally defined for integer superscript $n$ referring to the power of distance. We show, that most of the theory of Hansen coefficients remains valid for $X_{k}^{\gamma, j}$, when $\gamma$ is a real number. The generalized coefficients can be applied in a variety of perturbed problems that involve some drag effects. A more detailed discussion of the case $X_{0}^{(2 n+1) / 2, j}$ is given.
\end{abstract}

Key words: analytical methods, Hansen coefficients, Keplerian motion, special functions

\section{Introduction}

Hundred and fifty years since their invention, Hansen coefficients remain still one of the most important tools in the analytical methods of celestial mechanics. Following the classical summary given by Tisserand (1889), we define Hansen coefficients $X_{k}^{n, j}$ as the Fourier amplitudes in the series

$$
\left(\frac{r}{a}\right)^{n} \operatorname{expijf}=\sum_{k=-\infty}^{\infty} X_{k}^{n, j} \operatorname{expik} M,
$$

where $f$ is the true anomaly, $M$ is the mean anomaly, the ratio of the radial distance $r$ to the major semi-axis $a$ obeys the Keplerian formula

$$
\frac{r}{a}=\frac{1-e^{2}}{1+e \cos f}
$$

and we use the index $j \in \mathbb{N}$ instead of a traditional $m$. The negative values of $j$ are accounted for by the known symmetry $X_{k}^{n, j}=X_{-k}^{n,-j}$.

The theory of the coefficients $X_{n}^{k, j}$ as the functions of eccentricity $e$ or $\beta$

$$
\beta=\frac{e}{1+\sqrt{1-e^{2}}}
$$


has been worked out by many outstanding authors. The original paper by Hansen (1853) was followed by the contributions of Tisserand (1889), Newcomb (1895), von Zeipel (1912), Brumberg (1970), Aksenov (1986), and Giacaglia (1987) - to name just the few, without mentioning numerous articles that considered only computational aspects or the special cases known as the Kaula's eccentricity functions (Kaula, 1966).

The present paper stems from an observation, that the traditional assumption of an integer-valued $n$ in $X_{k}^{n, j}$ is not necessary: most of the fundamental formulae in the theory of Hansen coefficients admit a straightforward generalization to the case of

$$
\left(\frac{r}{a}\right)^{\gamma} \operatorname{expijf}=\sum_{k=-\infty}^{\infty} X_{k}^{\gamma, j} \operatorname{expi} k M,
$$

where $\gamma \in \mathbb{R}$. The generalization is more than just a mathematical curio; we have noticed it while averaging the Yarkovsky force perturbations (Breiter and Vokrouhlický, in preparation) and looking for some analogy with the Stokes drag perturbed motion. The latter problem was discussed by Ferraz-Mello (1992), and Jancart and Lemaître (2001), who applied some functions similar to the $X_{0}^{\gamma, j}$ coefficients with half-integer values of $\gamma$. For practical reasons, we focus our interest on the most useful subset $X_{0}^{\gamma, j}$ of the generalized Hansen coefficients that are required in analytical averaging. The general case $X_{k}^{\gamma, j}$ is only briefly discussed.

\section{Mean Value Related Hansen Coefficients}

Averaging Equation (1) with respect to the mean anomaly, one obtains the subset of Hansen coefficients

$$
\left\langle\left(\frac{r}{a}\right)^{n} \operatorname{expijf}\right\rangle=X_{0}^{n, j}
$$

with the subscript $k=0$. These classical functions (with $n \in \mathbb{Z}$ ) possess well known properties: they are expressible in terms of algebraic functions of eccentricity, they admit the reduction to the Gaussian hypergeometric series and hence they satisfy numerous linear recurrence relations (Aksenov, 1986). As we will see in the next sections, the last two properties hold true in the generalized case as well.

\subsection{HYPERGEOMETRIC SERIES FOR $X_{0}^{\gamma, j}$}

Suppressing the restriction on the values of $n$, we may consider

$$
X_{0}^{\gamma, j}=\frac{1}{\pi} \int_{0}^{\pi}\left(\frac{r}{a}\right)^{\gamma} \cos j f \mathrm{~d} M
$$


with a real superscript $\gamma$. Using the well-known Keplerian expressions (2) and

$$
\frac{\mathrm{d} f}{\mathrm{~d} M}=\eta\left(\frac{a}{r}\right)^{2}=\frac{(1+e \cos f)^{2}}{\eta},
$$

where $\eta=\sqrt{1-e^{2}}$, we can introduce a new, more convenient integration variable

$$
\phi=\frac{1}{2} f \text {. }
$$

Equation (6) takes the form

$$
\begin{aligned}
X_{0}^{\gamma, j} & =\frac{1}{\pi \eta} \int_{0}^{\pi}\left(\frac{r}{a}\right)^{\gamma+2} \cos j f \mathrm{~d} f \\
& =\frac{\eta^{2 \gamma+3}}{\pi} \int_{0}^{\pi} \frac{\cos j f}{(1+e \cos f)^{\gamma+2}} \mathrm{~d} f \\
& =\frac{2^{\gamma+2}}{\pi} \sqrt{2-m}\left(\frac{1-m}{2-m}\right)^{\gamma+3 / 2} \int_{0}^{\pi / 2} \frac{\cos (2 j \phi)}{\Delta^{2 \gamma+4}} \mathrm{~d} \phi,
\end{aligned}
$$

where

$$
\begin{aligned}
& \Delta=\sqrt{1-m \sin ^{2} \phi}, \\
& m=\frac{2 e}{1+e} .
\end{aligned}
$$

A quick look at Equation (9) reveals that the quadrature in the last line can be resolved as a closed form expression in terms of the complete elliptic integrals (Byrd and Friedman, 1954) for half-integer values of $\gamma$. Indeed, Ferraz-Mello (1992) or Jancart and Lemaittre (2001) considered the problem as an exercise in elliptic integrals. Starting with this important particular value of $\gamma$ we took this way at the beginning of our research, but then we noticed that - paradoxically the series expansion approach proves more attractive.

Observing, that $m<1$ in a non-degenerate elliptic motion, we can expand the integrand of Equation (9) in power series of $m$, obtaining

$$
X_{0}^{\gamma, j}=\frac{\sqrt{2-m}}{2^{2 j-\gamma-1}}\left(\frac{1-m}{2-m}\right)^{\gamma+3 / 2} \sum_{i=0}^{\infty} \frac{(-1)^{i}}{4^{i}}\left(\begin{array}{c}
-\gamma-2 \\
i+j
\end{array}\right)\left(\begin{array}{c}
2 i+2 j \\
i
\end{array}\right) m^{j+i} .
$$

The series (12) occurs to be related with the Gaussian hypergeometric function ${ }_{2} F_{1}$ (or simply $F$ ), and so

$$
\begin{aligned}
X_{0}^{\gamma, j}= & \left(-\frac{m}{4}\right)^{j}(1-m)^{\gamma+3 / 2}\left(\frac{2}{2-m}\right)^{\gamma+1} \frac{(\gamma+2)_{j}}{j !} \times \\
& \times F\left(\gamma+j+2, j+\frac{1}{2} ; 2 j+1 ; m\right),
\end{aligned}
$$


where we introduced Pochhammer symbol

$$
(a)_{b}=\frac{\Gamma(a+b)}{\Gamma(a)} .
$$

An elementary transformation leads to

$$
\begin{aligned}
X_{0}^{\gamma, j}= & \left(-\frac{m}{4}\right)^{j}\left(\frac{2}{2-m}\right)^{\gamma+1} \frac{(\gamma+2)_{j}}{j !} \times \\
& \times F\left(j-\gamma-1, j+\frac{1}{2} ; 2 j+1 ; m\right) .
\end{aligned}
$$

The hypergeometric function that occured in Equation (15) belongs to a 'friendly' class $F(\alpha, \beta ; 2 \beta ; m)$ that allows a quadratic transformation of argument (Gradshtein and Ryzhik, 1965)

$$
F(\alpha, \beta ; 2 \beta ; m)=\left(1-\frac{m}{2}\right)^{-\alpha} F\left(\frac{\alpha}{2}, \frac{\alpha+1}{2} ; \frac{2 \beta+1}{2} ;\left(\frac{m}{2-m}\right)^{2}\right) .
$$

Hence, noting that $[m /(2-m)]^{2}=e^{2}$, we can rewrite $X_{0}^{\gamma, j}$ using a better behaving hypergeometric function

$$
X_{0}^{\gamma, j}=\left(-\frac{e}{2}\right)^{j} \frac{(\gamma+2)_{j}}{j !} F\left(\frac{j-\gamma-1}{2}, \frac{j-\gamma}{2} ; j+1 ; e^{2}\right) .
$$

Thus we recover the classical expression of $X_{0}^{n, j}$ (Tisserand, 1889), but with an integer $n$ replaced by a real $\gamma$.

\subsection{SOME GENERAL PROPERTIES}

The hypergeometric function is a well-known mathematical object (Gradshtein and Ryzhik, 1965; Abramowitz and Stegun, 1972; Whittaker and Watson, 1990), hence Equations (15) and (17) allow to compute the $X_{0}^{\gamma, j}$ functions efficiently by means of standard software; they also furnish a multitude of formal identities, some of which will be discussed below.

\subsubsection{Symmetry}

While the symmetry of $X_{0}^{\gamma, j}$ in the $j$ index is trivial, and has been noted above, the relation between $X_{0}^{\gamma, j}$ coefficients with different values of $\gamma$ is less obvious, but it follows from the transformation properties of hypergeometric functions. If we replace $\gamma$ by $-\gamma-3$ in Equation (13), compare it with Equation (15) and note, that $(-\gamma-1)_{j}=(-1)^{j}(\gamma+2-j)_{j}$, we obtain

$$
X_{0}^{\gamma, j}=(-1)^{j} \eta^{2 \gamma+3} \frac{(\gamma+2)_{j}}{(\gamma+2-j)_{j}} X_{0}^{-\gamma-3, j}
$$

By virtue of this relation, functions $X_{0}^{\gamma, j}$ with $\gamma>-3 / 2$ can be expressed by those with $\gamma<-3 / 2$. Of course, the formula remains valid for $\gamma=n$ and - to 
our knowledge - it has not been reported earlier ${ }^{1}$ (probably due to a non-obvious 'turning point' $-3 / 2$ ). In the integer case, however, the validity of Equation (18) or of its inverse is restricted to the situations, when $(n+2-j)_{j} \neq 0$ or $(n+2)_{j} \neq 0$ respectively.

\subsubsection{Recurrence relations and derivative}

It was already Gauss who established the set of nine independent linear recurrence relations between the three neighboring hypergeometric functions. All known recurrence relations for $X_{0}^{n, j}$ follow directly from this set and can be easily generalized for real $\gamma$. We merely quote two of them

$$
X_{0}^{\gamma, j}=\frac{e}{2 j}\left[(\gamma+1-j) X_{0}^{\gamma, j+1}-(\gamma+1+j) X_{0}^{\gamma, j-1}\right],
$$

and

$$
X_{0}^{\gamma, j}=\frac{2 \gamma+1}{\gamma+1} X_{0}^{\gamma-1, j}-\frac{\gamma^{2}-j^{2}}{\gamma(\gamma+1)} \eta^{2} X_{0}^{\gamma-2, j},
$$

that are capable of generating all possible $X_{0}^{\gamma+n, j}$ coefficients with a given value of $\gamma \in \mathbb{R}$ and $n, j \in \mathbb{Z}$. All we need to start the process are, in principle 2 , but more conveniently four departure coefficients, say $X_{0}^{\{\gamma, \gamma+1\},\{0,1\}}$.

If the eccentricity is small, Equation (19) is not a useful numerical tool for computing $X_{0}^{\gamma, j+1}$, because it involves the division by $e$ with a potential loss of significant digits. In that case, however, hypergeometric series converge fast and they can be used more efficiently than recurrence formulas.

Arbitrary order derivatives of a hypergeometric function can be expressed as a hypergeometric function with shifted coefficients, times a numeric constant (Abramowitz and Stegun, 1972). Thanks to this property, the derivatives of $X_{0}^{\gamma, j}$ can always be expressed in terms of other Hansen coefficients. For example,

$$
\frac{\mathrm{d} X_{0}^{\gamma, j}}{\mathrm{~d} e}=\frac{j}{e} X_{0}^{\gamma, j}-\frac{(\gamma+1-j)(\gamma-j)}{\gamma+1} X_{0}^{\gamma-1, j+1} .
$$

Thus all classical expressions for the derivatives of $X_{0}^{n, j}$ can be generalized directly to the case of a real superscript.

\subsection{SPECIAL CASE WiTH $\gamma=(2 n+1) / 2$}

If $\gamma$ takes half-integer values, say $\gamma=(2 n+1) / 2$, with $n \in \mathbb{Z}$, all coefficients $X_{0}^{\gamma, j}$ can be expressed as a combination of algebraic functions of $e$ and of the complete elliptic integrals of the first and the second kind $\mathbf{K}(m), \mathbf{E}(m)$. This can be seen if we specify four initial coefficients. Let use Equation (15) with $\gamma \in\{-3 / 2,-1 / 2\}$ $n \in \mathbb{N}$.

${ }_{\mathbb{N}}^{1}$ Hughes (1981) still used two different recursive algorithms for $X_{0}^{n, j}$ and $X_{0}^{-(n+1), j}$ with 
and $j \in\{0,1\}$. Then we obtain

$$
\begin{aligned}
X_{0}^{-3 / 2,0} & =\left(\frac{2}{2-m}\right)^{-1 / 2} F\left(\frac{1}{2}, \frac{1}{2} ; 1 ; m\right)=\frac{2}{\pi} \frac{\mathbf{K}(m)}{\sqrt{1+e}} \\
X_{0}^{-1 / 2,0} & =\left(\frac{2}{2-m}\right)^{1 / 2} F\left(-\frac{1}{2}, \frac{1}{2} ; 1 ; m\right)=\frac{2}{\pi} \sqrt{1+e} \mathbf{E}(m) \\
X_{0}^{-3 / 2,1} & =\frac{m}{8}\left(\frac{2}{2-m}\right)^{-1 / 2} F\left(\frac{3}{2}, \frac{3}{2} ; 3 ; m\right) \\
& =-\frac{2}{\pi} \frac{\mathbf{K}(m)-(1+e) \mathbf{E}(m)}{e \sqrt{1+e}} \\
X_{0}^{-1 / 2,1} & =-\frac{m}{8}\left(\frac{2}{2-m}\right)^{1 / 2} F\left(\frac{1}{2}, \frac{3}{2} ; 3 ; m\right) \\
& =-\frac{2}{\pi} \frac{\sqrt{1+e}}{e}[\mathbf{E}(m)-(1-e) \mathbf{K}(m)]
\end{aligned}
$$

where, as usually, $m=2 e /(1+e)$. Equations (19) and (20) imply, that starting from the above initial coefficients, we will always obtain

$$
X_{0}^{(2 n+1) / 2, j}=F_{1}(e) \mathbf{E}(m)+F_{2}(e) \mathbf{K}(m),
$$

where both $F_{1}$ and $F_{2}$ are algebraic functions of $e$.

Interestingly, if the Gaussian transformation of modulus is applied (Abramowitz and Stegun, 1972), we can also use the elliptic integrals with a modulus $\beta^{2}$, because

$$
m=\frac{4 \beta}{(1+\beta)^{2}}
$$

and so

$$
\begin{aligned}
& \mathbf{K}(m)=(1+\beta) \mathbf{K}\left(\beta^{2}\right), \\
& \mathbf{E}(m)=\frac{1}{1+\beta}\left[2 \mathbf{E}\left(\beta^{2}\right)-\left(1-\beta^{2}\right) \mathbf{K}\left(\beta^{2}\right)\right] .
\end{aligned}
$$

Of course, the same result could be achieved by a transformation of the hypergeometric function. ${ }^{2}$

\subsection{ON A RELATED FUNCTION $Y_{0}^{\gamma, j}$}

In some situations one may prefer the use of eccentric anomaly $u$; in that case the classical results can be found in (Ahmed, 1994). So, let us consider also a function

$$
Y_{0}^{\gamma, j}=\frac{1}{\pi} \int_{0}^{\pi}\left(\frac{r}{a}\right)^{\gamma} \cos j u \mathrm{~d} M,
$$

\footnotetext{
${ }^{2}$ cf. Equation $\left(D_{1}\right)$ in Tisserand (1889, p. 255).
} 
just in order to show, that it can be easily related to $X_{0}^{\gamma, j}$ and thus no special theory is required for a 'quasi-Hansen coefficient' $Y_{0}^{\gamma, j}$.

Following a similar reasoning as above and using:

$$
\frac{\mathrm{d} u}{\mathrm{~d} M}=\frac{a}{r}=\frac{1}{1-e \cos u},
$$

we obtain

$$
\begin{aligned}
Y_{0}^{\gamma, j} & =\frac{1}{\pi} \int_{0}^{\pi}\left(\frac{r}{a}\right)^{\gamma+1} \cos j u \mathrm{~d} u \\
& =\frac{1}{\pi} \int_{0}^{\pi}(1-e \cos u)^{\gamma+1} \cos j u \mathrm{~d} u \\
& =(-1)^{j} \frac{2}{\pi}\left(\frac{2}{2-m}\right)^{\gamma+1} \int_{0}^{\pi / 2} \Delta^{2 \gamma+2} \cos (2 j \phi) \mathrm{d} \phi,
\end{aligned}
$$

where $\phi=u / 2$. Expanding $\Delta$ in powers of $m$ and using the transformation properties of the hypergeometric functions we finally have

$$
Y_{0}^{\gamma, j}=\left(-\frac{e}{2}\right)^{j} \frac{(\gamma+2-j)_{j}}{j !} F\left(\frac{j-\gamma-1}{2}, \frac{j-\gamma}{2} ; j+1 ; e^{2}\right) .
$$

Comparison with Equation (17) then yields a direct and simple relation

$$
Y_{0}^{\gamma, j}=\frac{(\gamma+2-j)_{j}}{(\gamma+2)_{j}} X_{0}^{\gamma, j}
$$

As we see, both functions are equivalent up to a numeric factor.

\section{Hansen Coefficients With Arbitrary $k$}

The theory of $X_{k}^{\gamma, j}$ with $k \neq 0$ is more complicated, because the coefficients are no longer directly related to the hypergeometric function ${ }_{2} F_{1}$. In the present paper, we have only checked which of the known formulae for the direct computation of $X_{k}^{n, j}$ admit a straightforward generalization for $X_{k}^{\gamma, j}$. In each case we compared the formula with a numerical quadrature

$$
X_{k}^{\gamma, j}=\frac{1}{2 \pi} \int_{-\pi}^{\pi}\left(\frac{r}{a}\right)^{\gamma} \exp (\mathrm{i} k f) \exp (-\mathrm{i} j M) \mathrm{d} M .
$$

The Tisserand's formula (Tisserand, 1889; Zarouatti, 1987) becomes simply

$$
X_{k}^{\gamma, j}=\frac{(-\beta)^{|k-j|}}{\left(1+\beta^{2}\right)^{\gamma+1}} \sum_{s=0}^{\infty} \mathcal{P}_{s} \mathcal{Q}_{s} \beta^{2 s},
$$


where

$$
\begin{aligned}
& \mathcal{P}_{s}= \begin{cases}P_{s+k-j} & \text { if } k \geqslant j, \\
P_{s} & \text { if } k<j,\end{cases} \\
& \mathcal{Q}_{s}= \begin{cases}Q_{j} & \text { if } k \geqslant j, \\
Q_{s+j-k} & \text { if } k<j,\end{cases} \\
& P_{s}=\sum_{r=0}^{s}\left(\begin{array}{c}
\gamma-j+1 \\
s-r
\end{array}\right) \frac{1}{r !}\left(-\frac{k e}{2 \beta}\right)^{r}, \\
& Q_{s}=\sum_{r=0}^{s}\left(\begin{array}{c}
\gamma+j+1 \\
s-r
\end{array}\right) \frac{1}{r !}\left(\frac{k e}{2 \beta}\right)^{r} .
\end{aligned}
$$

Among the numerous (although essentially equivalent) variants of Bessel function series for $X_{k}^{n, j}$ (Jarnagin, 1965; Giacaglia, 1976, 1987) we may recommend the form given by Aksenov $(1986)^{3}$ that can be directly taken as

$$
X_{k}^{\gamma, j}=\left(1+\beta^{2}\right)^{-\gamma-1} \sum_{s=-\infty}^{\infty} E_{k-s}^{\gamma, j} J_{s}(k e),
$$

where

$$
\begin{aligned}
E_{p}^{\gamma, j}= & (-\beta)^{p-j}\left(\begin{array}{c}
\gamma-j+1 \\
p-j
\end{array}\right) \times \\
& \times F\left(-\gamma-j-1, p-\gamma-1 ; p-j+1 ; \beta^{2}\right) \quad \text { for } p \geqslant j,
\end{aligned}
$$

and $E_{p}^{\gamma, j}=E_{-p}^{\gamma,-j}$ should be applied for $p<j$.

It is worth noting, that if $\gamma=(2 n+1) / 2$, the coefficients $E_{p}^{\gamma, j}$ will have a generic form similar to Equation (26), involving either the elliptic integrals with modulus $\beta^{2}$ or (after the Gauss transformation) with modulus $m$.

\section{Conclusions}

Hansen coefficients can be easily generalized to include any power of the distance $r^{\gamma}$. Thus, no new theory is required for the elliptic expansions in Stokes drag perturbed problems or in other problems involving the drag proportional to more exotic powers of velocity and distance. Hansen's assumption of integer $n$ was justified by the most common application domain of $X_{k}^{n, j}$ coefficients (i.e. gravitational perturbations), but it can be quite easily suppressed. Most of the known formulae admit a straightforward extension to the real $\gamma$ case, save for the ones that make use of the property, that hypergeometric series with negative integer arguments

${ }^{3}$ See also Branham (1990). 
terminate. The theory of hypergeometric functions was present in the Hansen coefficients problems since the beginning and - in our opinion - it still remains the most handy tool in this domain, unless some FFT-like algorithms are applied (see Brumberg and Brumberg, 1999).

\section{References}

Abramowitz, M. and Stegun, I. A.: 1972, Handbook of Mathematical Functions with Formulas, Graphs, and Mathematical Tables, Wiley-Interscience, New York.

Ahmed, M. K. M.: 1994, 'On the normalization of perturbed Keplerian systems', Astron. J. 107, 1900-1903.

Aksenov, E. P.: 1986, Special Functions in Celestial Mechanics, Nauka, Moscow (in Russian).

Branham, R. L.: 1990, 'Recursive calculation of Hansen coefficients', Celest. Mech. \& Dyn. Astr. 49, 209-217.

Brumberg, V. A.: 1970, 'On a differential equation for Hansen coefficients', Bull. ITA 12, 452-457, (in Russian).

Brumberg, V. A. and Brumberg, E. V.: 1999, Celestial Dynamics at High Eccentricities, Gordon and Breach, Amsterdam.

Byrd, P. F. and Friedman, M. D.: 1954, Handbook of Elliptic Integrals for Engineers and Physicist, Springer, Berlin.

Ferraz-Mello, S.: 1992, 'Averaging the elliptic asteroidal problem with a Stokes drag', In: Interrelations Between Physics and Dynamics for Minor Bodies in the Solar System. Gif-sur-Yvette, Frontières, pp. 45-60.

Giacaglia, G. E. O.: 1976, 'A note on Hansen's coefficients in satellite theory', Celest. Mech. 14, $515-523$.

Giacaglia, G. E. O.: 1987, 'Hansen coefficients and generalized spherical harmonics', Publ. Astron. Soc. Jpn. 39, 171-178.

Gradshtein, I. S. and Ryzhik, I. M.: 1965, Tables of Series, Products and Integrals, Academic Press, New York.

Hansen, P. A.: 1853, 'Entwickelung des Products einer Potenz des Radius Vector mit dem Sinus oder Cosinus eines Vielfachen der wahren Anomalie in Reihen', Abhandl. d. K. S. Ges. d. Wissensch. 2, 183-281.

Hughes, S.: 1981, 'The computation of tables of Hansen coefficients', Celest. Mech. 25, 101-107.

Jancart, S. and Lemaître, A.: 2001, 'Dissipative forces and external resonances', Celest. Mech. \& Dyn. Astr. 81, 75-80.

Jarnagin, M. P.: 1965, 'Expansions in elliptic motion', Astr. Pap. 18, 36.

Kaula, W. M.: 1966, Theory of Satellite Geodesy. Blaisdell Publ. Co., Waltham.

Newcomb, S.: 1895, 'Development of the perturbative function in cosines of multiples of the mean anomalies and of angles between the perihelia and common node and in powers of the eccentricities and mutual inclinations', Astr. Pap. 5, 1-48.

Tisserand, F.: 1889, Traité de Mécanique Céleste, Gauthier-Villars, Paris.

von Zeipel, H.: 1912, 'Entwicklung der Störungsfunktion', In: Encyklopädie der Mathematische Wissenschaften, Bd VI 2. Leipzig, Teubner, pp. 557-665.

Whittaker, E. T. and Watson, G. N.: 1990, A Course in Modern Analysis, 4th edn, Cambridge University Press, Cambridge, 281-301.

Zarouatti, O.: 1987, Trajectoires Spatiales, Cepadues-Editions, Toulouse, 207-209. 